\title{
New Fast Algorithm for Modulated Complex Lapped Transform With Sine Windowing Function
}

\author{
Huazhong Shu, Senior Member, IEEE, Jiasong Wu, Lotfi Senhadji, Senior Member, IEEE, and
} Limin Luo, Senior Member, IEEE

\begin{abstract}
A novel algorithm for fast computation of the modulated complex lapped transform (MCLT) with sine windowing function is presented. For the MCLT of length-2 $M$ input data sequence, the proposed algorithm is based on computing a length-2 $M$ type-II generalized discrete Hartley transform. Comparison with existing algorithms shows that the proposed method achieves the minimal number of arithmetic operations.
\end{abstract}

Index Terms-Audio processing, discrete Hartley transform, fast algorithm, lapped transform.

\section{INTRODUCTION}

$\mathbf{T}$ HE modulated complex lapped transform (MCLT) is a cosine-modulated filter bank that maps overlapping blocks of a real-valued signal into complex-valued blocks of transform coefficients [1]. The MCLT is a complex extension of the modulated lapped transform (MLT) introduced by Malvar [2], and the latter transform is equivalent to the modified discrete cosine transform (MDCT) introduced in [3]. The real part of the MCLT corresponds to the MLT of the same signal, and the imaginary part carries phase information. Thus, the MCLT can be used in applications where the magnitude-phase decomposition is required, as well as interfacing to coding systems such as in audio watermarking [4] and identification [5]. The advantage of the MCLT over the discrete Fourier transform (DFT) filter banks is that the reconstruction formula of MCLT is not unique, so that it allows more flexible implementation of audio enhancement and encoding system.

Several fast algorithms for computing the MCLT have been reported in the literature. Malvar [1] suggested an efficient way for calculating the MCLT in which the cosine component is evaluated via an MLT algorithm based on the type-IV discrete cosine

This work was supported in part by the National Natural Science Foundation of China under Grant 60873048, in partby the Program for Changjiang Scholar and Innovative Research Team in University, and in part by the Natural Science Foundation of Jiangsu Province under Grant BK2008279.

H. Shu, J. Wu, and L. Luo are with the Laboratory of Image Science and Technology, School of Computer Science and Engineering, Southeast University, 210096 Nanjing, China, and also with the Centre de Recherche en Information Biomédicale Sino-Français (CRIBs) (e-mail: shu.list@ seu.edu.cn; jswu@seu. edu.cn; luo.list@seu.edu.cn).

L. Senhadji is with the INSERM, U642, Rennes 35042, France, with the Université de Rennes 1, LTSI, Rennes 35042, France, and also with the Centre de Recherche en Information Biomédicale Sino-Français (CRIBs) (e-mail: lotfi. senhadji@univ-rennes1.fr). transform (DCT-IV), and the sine component via a slightly modified MLT algorithm based on the type-IV discrete sine transform. Malvar subsequently proposed a fast MCLT algorithm of sequence length- $2 M$ via the computation of length- $2 M$ DFT [6]. The DFT-based algorithm not only reduces the computational complexity compared to the algorithm presented in [1] but also does not require data shuffling. Dai and Chen [7] developed a DCT-based algorithm for the efficient computation of the MCLT, and their algorithm is applicable to any symmetrical windowing function. For a sine window, Dai's algorithm requires slightly more number of arithmetic operations than that of the algorithm reported in [6]. The DCT-based algorithm was further improved by the same authors [8]. Other MCLT algorithm for purpose of hardware implementation was proposed by Tai and Jing [9].

In this letter, we propose a new algorithm for computing the MCLT with sine windowing function. The algorithm is based on the type-II generalized discrete Hartley transform (GDHT-II) introduced by $\mathrm{Hu}$ et al. [10], which is equivalent to the generalized discrete $\mathrm{W}$ transform introduced in [11]. For an MCLT of length- $2 M$ input data sequence, both real and imaginary parts of the MCLT coefficients are calculated via a length-2 $M$ GDHT-II. Compared to previous algorithms, the proposed one achieves the lowest computational complexity.

\section{Definition And New Fast Algorithm}

The MCLT of length- $2 M$ real input data sequence $\{x(n)\}$, $n=0,1, \ldots, 2 M-1$ is defined by [1]

$$
X(k)=\sum_{n=0}^{2 M-1} x(n) p(n, k), \quad k=0,1, \ldots, M-1
$$

where $p(n, k)$ is the analysis basis function defined by $p(n, k)=$ $p_{c}(n, k)-j p_{s}(n, k)$ with $j=\sqrt{-1}$ and

$$
\begin{aligned}
& p_{c}(n, k)=\sqrt{\frac{2}{M}} h(n) \cos \left[\frac{\pi}{4 M}(2 n+1+M)(2 k+1)\right] \\
& p_{s}(n, k)=\sqrt{\frac{2}{M}} h(n) \sin \left[\frac{\pi}{4 M}(2 n+1+M)(2 k+1)\right] .
\end{aligned}
$$

Here $h(n)$ is the windowing function. A common choice of $h(n)$ is the symmetrical sine windowing function given by

$$
h(n)=-\sin \frac{\pi(2 n+1)}{4 M}, \quad h(n)=h(2 M-1-n) .
$$

Equation (1) can be rewritten as

$$
X(k)=X_{c}(k)-j X_{s}(k)
$$


where $X_{c}(k)$ and $X_{s}(k)$ are, respectively, the real part and imaginary part of $X(k)$, defined by

$$
\begin{aligned}
& X_{c}(k)=\sum_{n=0}^{2 M-1} x(n) p_{c}(n, k) \\
& X_{s}(k)=\sum_{n=0}^{2 M-1} x(n) p_{s}(n, k), \quad k=0,1, \ldots, M-1 .
\end{aligned}
$$

The backward MCLT can be calculated via the following reconstruction formula [1]:

$$
\begin{aligned}
& \widetilde{x}(n)=\beta_{c} \sum_{k=0}^{M-1} X_{c}(k) p_{c}(n, k)+\beta_{s} \sum_{k=0}^{M-1} X_{s}(k) p_{s}(n, k) \\
& n=0,1, \ldots, 2 M-1
\end{aligned}
$$

where the coefficients $\beta_{c}$ and $\beta_{s}$ are chosen as $\beta_{c}+\beta_{s}=1$.

We now propose a new fast algorithm for computing the MCLT coefficients $X(k)$ given by (4). From (2a) and (2b), it can be easily verified that

$$
\begin{aligned}
p_{c}(n, 2 M-1-k)= & (-1)^{M+1} p_{c}(n, k) \\
p_{s}(n, 2 M-1-k)= & (-1)^{M} p_{s}(n, k) \\
& k=0,1, \ldots, M-1 .
\end{aligned}
$$

So that

$$
\begin{aligned}
X_{c}(2 M-1-k)= & (-1)^{M+1} X_{c}(k) \\
X_{s}(2 M-1-k)= & (-1)^{M} X_{s}(k) \\
& k=0,1, \ldots, M-1 .
\end{aligned}
$$

The above equations imply that $X_{c}(k)$ and $X_{s}(k)$ satisfy even antisymmetry and symmetry property, respectively, depending on the parity of $M$. Therefore, the computation of $X_{c}(k)$ and $X_{s}(k)$ for $k=0,1, \ldots, M-1$ can be done by computing either the even-indexed or odd-indexed coefficients $X(k)$ for $k$ varying from 0 to $2 M-1$. We choose here the even-indexed coefficients, and (2a) becomes

$$
\begin{aligned}
p_{c}(n, 2 k)= & \sqrt{\frac{2}{M}} h(n) \cos \left[\frac{\pi}{4 M}(2 n+1+M)(4 k+1)\right] \\
= & -\sqrt{\frac{2}{M}} \sin \frac{(2 n+1) \pi}{4 M} \\
& \times \cos \left[\frac{\pi}{4}(4 k+1)+\frac{\pi}{4 M}(2 n+1)(4 k+1)\right] \\
= & -(-1)^{k} \sqrt{\frac{1}{M}} \sin \frac{(2 n+1) \pi}{4 M} \\
& \times \operatorname{cas} \frac{-\pi(2 n+1)(4 k+1)}{4 M} \\
& k=0,1, \ldots, M-1
\end{aligned}
$$

where $\operatorname{cas} \theta=\cos \theta+\sin \theta$.

The above derivation is similar to one result reported in [12] where the authors have established a relationship between the MDCT and GDHT-IV. For the windowing function $h(n)$ defined by (3), applying the trigonometric property

$$
-\sin (\alpha) \operatorname{cas}(-\beta)=\frac{1}{2}[\cos (\beta-\alpha)-\operatorname{cas}(\alpha+\beta)]
$$

(9) becomes

$$
\begin{aligned}
p_{c}(n, 2 k) & =\frac{(-1)^{k}}{2} \sqrt{\frac{1}{M}}\left[\operatorname{cas} \frac{\pi(2 n+1) 2 k}{2 M}\right. \\
& \left.-\operatorname{cas} \frac{\pi(2 n+1)(2 k+1)}{2 M}\right], \quad k=0,1, \ldots, M-1 .
\end{aligned}
$$

Similarly

$$
\begin{aligned}
p_{s}(n, 2 k)= & \sqrt{\frac{2}{M}} h(n) \sin \left[\frac{\pi}{4 M}(2 n+1+M)(4 k+1)\right] \\
= & -\sqrt{\frac{2}{M}} \sin \frac{(2 n+1) \pi}{4 M} \\
& \times \sin \left[\frac{\pi}{4}(4 k+1)+\frac{\pi}{4 M}(2 n+1)(4 k+1)\right] \\
= & -(-1)^{k} \sqrt{\frac{1}{M}} \sin \frac{(2 n+1) \pi}{4 M} \\
& \times \operatorname{cas} \frac{\pi(2 n+1)(4 k+1)}{4 M} \\
= & \frac{(-1)^{k}}{2} \sqrt{\frac{1}{M}}\left[\operatorname{cas} \frac{-\pi(2 n+1)(2 k+1)}{2 M}\right. \\
& k=0,1, \ldots, M-1 .
\end{aligned}
$$

Letting

$$
\begin{aligned}
& Y(k)= \sqrt{\frac{1}{2 M}} \sum_{n=0}^{2 M-1} x(n) \operatorname{cas} \frac{\pi(2 n+1) k}{2 M} \\
& Z(k)=\sqrt{\frac{1}{2 M}} \sum_{n=0}^{2 M-1} x(n) \operatorname{cas} \frac{-\pi(2 n+1) k}{2 M} \\
& k=0,1, \ldots, 2 M-1
\end{aligned}
$$

it can be easily verified that

$$
Z(2 M-k)=-Y(k), \quad k=0,1, \ldots, 2 M-1 .
$$

Substituting (11) and (12) into (5a) and (5b), and using (13)-(15), we obtain

$$
\begin{aligned}
X_{c}(2 k) & =\frac{(-1)^{k}}{\sqrt{2}}[Y(2 k)-Y(2 k+1)] \\
X_{s}(2 k) & =\frac{(-1)^{k}}{\sqrt{2}}[Z(2 k+1)-Z(2 k)] \\
& =\frac{(-1)^{k}}{\sqrt{2}}[Y(2 M-2 k)-Y(2 M-2 k-1)]
\end{aligned}
$$

for $k=0,1, \ldots, M-1$. Notice that $Y(2 M)=-Y(0)$.

It is worth noting that $Y(k)$ defined by (13) is a normalized length-2 $M$ GDHT-II of the input data sequence $x(n)$;thus, it can be computed via FHT algorithm. When the values of $Y(k)$, $k=0,1, \ldots, 2 M-1$ are available, the real part and imaginary part of MCLT coefficients, $X_{c}(2 k)$ and $X_{s}(2 k)$, for $k=$ $0,1, \ldots, M-1$, can be easily obtained from (16a) and (16b). Once the even-indexed coefficients are obtained, the odd-indexed coefficients can be deduced from the symmetry property (8a) and (8b). The flowgraph realizing the proposed algorithm 


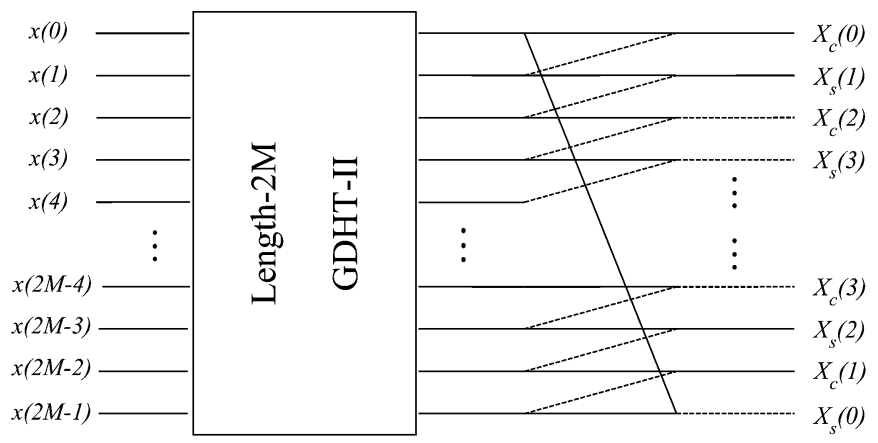

Fig. 1. Fowgraph of the proposed algorithm for even value of $M$. Solid lines denote unity transfer factor, and dot lines denote transfer factor -1 . $\bullet$ denotes adder.

is shown in Fig. 1. The flowgraph for the corresponding backward MCLT can be easily inferred from this figure.

The above discussion shows that the computational complexity of the proposed algorithm is that of a length- $2 M$ GDHT-II, plus the arithmetic operations in (16a) and (16b). Since many FHT algorithms are available [13], [14], they can be used to improve the computational efficiency. For $M$ being a power of two, using the algorithm reported in [14], the length-2 $M$ GDHT-II requires $(4 / 3) M \log _{2} M-(8 / 9) M-(10 / 9)(-1)^{\log _{2} M}$ multiplications and $(8 / 3) M \log _{2} M+(8 / 9) M+(10 / 9)(-1)^{\log _{2} M}$ additions where the butterfly computations are implemented by four multiplications and two additions (4mult-2add scheme). Because the implementation of the butterfly computation can also be realized with three multiplications and three additions (3mult-3add scheme), in such a case, Bi's algorithm for computing the length-2M GDHT-II needs $M \log _{2} M-(1 / 3) M-(2 / 3)(-1)^{\log _{2} M}$ multiplications and $3 M \log _{2} M+(1 / 3) M+(2 / 3)(-1)^{\log _{2} M}$ additions. Besides the FHT, we need to perform $2 M$ additions involved in (16a) and (16b). Because the factor $1 / \sqrt{2}$ can be either absorbed into the scaling stage of GDHT or into the quantization stage in coding application, no multiplication is required in (16a) and (16b). Thus, the total number of arithmetic operations of the proposed method is $(8 / 3) M \log _{2} M+(26 / 9) M+(10 / 9)(-1)^{\log _{2} M}$ additions and $(4 / 3) M \log _{2} M-(8 / 9) M-(10 / 9)(-1)^{\log _{2} M}$ multiplications if the 4mult-2add scheme is considered, and $3 M \log _{2} M+(7 / 3) M+(2 / 3)(-1)^{\log _{2} M}$ additions and $M \log _{2} M-(1 / 3) M-(2 / 3)(-1)^{\log _{2} M}$ multiplications if the 3 mult-3add scheme is applied. For the case where $M$ is not a power of two, the length-2 $M$ GDHT-II can be calculated via the fast algorithms reported in [13]-[15].

The comparison of the proposed algorithm with other algorithms in terms of the computational complexity for $M$ being a power of two is listed in Table I. It can be seen from this table that for the common sine windowing function, our algorithm requires not only the least number of multiplications if the 3mult-3add scheme is used for implementing the butterfly computation but also the least number of additions among all the existing MCLT algorithms. In the algorithms reported in [7] and [8], the authors converted the real part and imaginary part of the $2 M$-point MCLT coefficients into two $M$-point DCT-II coefficients by absorbing the sine windowing function into the
TABLE I

COMPUTATIONAl COMPLEXITY OF SEVERAL FAST MCLT ALGORITHMS FOR Block Size BeIng a Power of Two (* MeAns That the 4Mult-2AdD SCHEME Is Used, AND $* *$ MeAns That THE 3Mult-3AdD Scheme Is CONSIDERED)

\begin{tabular}{|c|c|c|c|}
\hline Algorithm & $\begin{array}{l}\text { Windowing } \\
\text { function } \\
\text { choice }\end{array}$ & Number of multiplications & Number of additions \\
\hline Ref. [1] & any & $M \log _{2} M+4 M$ & $3 M \log _{2} M+2 M$ \\
\hline Ref. [6] & sine & $M \log _{2} M+M$ & $3 M \log _{2} M+3 M-2$ \\
\hline \multirow[t]{2}{*}{ Ref. [7] } & $\begin{array}{c}\text { symmetrica } \\
1\end{array}$ & $M \log _{2} M+3 M$ & \multirow[t]{2}{*}{$3 M \log _{2} M+4 M$} \\
\hline & sine & $M \log _{2} M+M$ & \\
\hline \multirow[t]{2}{*}{ Ref. $[8]$} & any & $M \log _{2} M+2 M$ & \multirow[t]{2}{*}{$3 M \log _{2} M+4 M$} \\
\hline & sine & $M \log _{2} M$ & \\
\hline Ref. [9] & any & $2 M \log _{2} M+3 M+2$ & $6 M \log _{2} M+3 M+4$ \\
\hline \multirow{2}{*}{ Proposed } & \multirow{2}{*}{ sine } & $* \frac{4}{3} M \log _{2} M-\frac{8}{9} M-\frac{10}{9}(-1)^{\log _{2} M}$ & $* \frac{8}{3} M \log _{2} M+\frac{26}{9} M+\frac{10}{9}(-1)^{\log _{2} M}$ \\
\hline & & ${ }^{* *} M \log _{2} M-\frac{1}{3} M-\frac{2}{3}(-1)^{\log _{2} M}$ & $* * 3 M \log _{2} M+\frac{7}{3} M+\frac{2}{3}(-1)^{\log _{2} M}$ \\
\hline
\end{tabular}

kernel function of DCT-IV. In our algorithm, we have converted the real part and imaginary part of the $2 M$-point MCLT coefficients into the computation of a $2 M$-point GDHT-II coefficients by absorbing the sine windowing function into the kernel function of GDHT-IV. It should be pointed out that the algorithms presented in [7] and [8] can be applied, respectively, to the symmetrical windowing function and any windowing function. However, our algorithm seems only to support the sine windowing function.

\section{CONCLUSION}

In this letter, a new algorithm for the efficient computation of the MCLT with sine windowing function is presented. For an MCLT of length- $2 M$ input data sequence, both the real part and imaginary part of the MCLT coefficients can be obtained from the length-2 $M$ GDHT-II coefficients of the same signal. The proposed algorithm achieves the least number of real multiplications and the least total number of arithmetic operations, compared to previous algorithms, with saving on the order of $5 \%$. Moreover, the proposed method has regular structure, so it can be useful for both software and hardware implementations.

\section{REFERENCES}

[1] H. S. Malvar, "A modulated complex lapped transform and its application to audio processing," in Proc. ICASSP, Phoenix, AZ, Mar. 1999, pp. 1421-1424.

[2] H. S. Malvar, Signal Processing With Lapped Transform. Boston, MA: Artech House, 1992.

[3] J. P. Princen, A. W. Johnson, and A. B. Bradley, "Subband/transform coding using filter bank design based on time domain aliasing cancellation," in Proc. IEEE ICASSP'87, Dallas, TX, 1987, pp. 2161-2164.

[4] D. Kirovski and H. Malvar, "Robust spread-spectrum audio watermarking," in Proc. ICASSP, Salt Lake City, UT, Apr. 2001, pp. $1345-1348$

[5] C. J. C. Burges, J. C. Platt, and J. Jana, "Extracting noise-robust features from audio data," in Proc. ICASSP, Orlando, FL, May 2002, pp. 1021-1024.

[6] H. S. Malvar, "Fast algorithm for the modulated complex lapped transform," IEEE Signal Process. Lett., vol. 10, no. 1, pp. 8-10, Jan. 2003. 
[7] Q. Dai and X. Chen, "New algorithm for modulated complex lapped transform with symmetrical window function," IEEE Signal Process. Lett., vol. 11, no. 12, pp. 925-928, Dec. 2004.

[8] X. Chen and Q. Dai, "A novel DCT-based algorithm for computing the modulated complex lapped transform," IEEE Trans. Signal Process. vol. 54, no. 11, pp. 4480-4484, Nov. 2006.

[9] H. M. Tai and C. Jing, "Design and efficient implementation of a modulated complex lapped transform processor using pipeline technique," IEICE Trans. Fundam., vol. E84-A, no. 5, pp. 1280-1286, 2001.

[10] N. C. Hu, H. I. Chang, and O. K. Ersoy, "Generalized discrete Hartley transforms," IEEE Trans. Signal Process., vol. 40, no. 12, pp. 2931-2940, Dec. 1992.

[11] Z. Wang, "Comments on generalized discrete Hartley transform," IEEE Trans. Signal Process., vol. 43, no. 7, pp. 1171-1172, Jul. 1995.
[12] V. Britanak and K. R. Rao, "A new fast algorithm for the unified forward and inverse MDCT/MDST computation," Signal Process., vol. 82, no. 3, pp. 433-459, 2002.

[13] V. Britanak and K. R. Rao, "The fast generalized discrete Fourier transforms: A unified approach to the discrete sinusoidal transform computation," Signal Process., vol. 79, no. 12, pp. 135-150, 1999.

[14] G. Bi, Y. Chen, and Y. Zeng, "Fast algorithms for generalized discrete Hartley transform of composite sequence lengths," IEEE. Trans. Circuits Syst. II, vol. 47, no. 9, pp. 893-901, Sep. 2000.

[15] G. Bi and Y. Chen, "Fast generalized DFT and DHT algorithms," Signal Process., vol. 65, no. 3, pp. 383-390, 1998. 